\title{
Molecular Wires in Electromagnetic Fields
}

\author{
Sigmund Kohler, Jörg Lehmann, Michael Strass, and Peter Hänggi \\ Institut für Physik, Universität Augsburg, 86135 Augsburg, Germany
}

\begin{abstract}
We investigate the role of external electromagnetic fields on the conduction properties of bridged molecular wires. In particular, it is analyzed quantitatively how resonant excitations of electrons enhance the dc current and, simultaneously, lower the noise level of the current. The results from an exact numerical treatment are in good agreement with those obtained within an approximation scheme applicable at resonances.
\end{abstract}

Thirty years ago, Aviram and Ratner proposed in a seminal work [1] to build elements of electronic circuits - in their case a rectifier - with single molecules. In the present days their vision starts to become reality and the experimental and theoretical study of such systems enjoys a vivid activity [2-4]. Recent experimental progress has enabled reproducible measurements $[5,6]$ of weak tunneling currents through molecules which are coupled by chemisorbed thiol groups to the gold surface of external leads.

Typical energy scales in molecules are in the optical and the infrared regime, where basically all of the today's lasers operate. Hence, lasers represent a natural possibility to control atoms or molecules and also currents through them. It is for example possible to induce by the laser field an oscillating current in the molecule which under certain asymmetry conditions is rectified by the molecule. This results in a directed electron transport even in the absence of any applied voltage $[7,8]$. Another theoretically predicted effect is the current suppression by the laser field $[9,10]$ which offers the possibility to control both the average current and the current noise.

Since the considered frequencies lie below typical plasma frequencies of metals, the laser light will be reflected at the metal surface, i.e., it does not penetrate the leads. Consequently, we assume that the leads' bulk properties are essentially unaffected by the laser field - in particular each lead remains close to equilibrium. Thus, it is sufficient to consider the influence of the driving solely in the molecule Hamiltonian. In addition, the energy of infrared light quanta is by far smaller than the work function of a common metal, which is of the order of $5 \mathrm{eV}$. This prevents the generation of a photo current, which otherwise would dominate the effects discussed below. For a quantitative description of an experiment, it might be necessary to take into account also the influence of the laser on the leads.

Most theoretical descriptions of the molecular conductivity in static situations are based on a scattering approach [11-13], or assume that the un- 


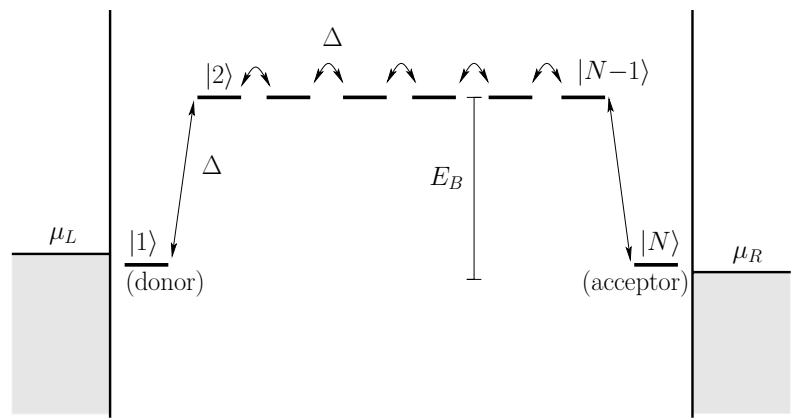

Fig. 1. Bridged molecular wire consisting of $N=8$ sites of which the first and the last site are coupled to leads with chemical potentials $\mu_{L}$ and $\mu_{R}-\mathrm{eV}$

derlying transport mechanism is an electron transfer reaction from the donor to the acceptor site and that the conductivity can be derived from the corresponding reaction rates [3].

Atoms and molecules in strong oscillating fields have been widely studied within a Floquet formalism [14,15]. This suggests utilizing the tools that have been acquired in that area, thus, developing a transport formalism that combines Floquet theory for a driven molecule with the many-particle description of transport through a system that is coupled to ideal leads $[8,10,16]$.

As an idealized model for the wire and the leads, we employ the so-called bridged molecule setup sketched in Fig. 1. This model has also been used to, e.g., investigate inelastic effects due to electron-vibrational coupling [17-20] and heat conduction [21]. A central issue in these works has been the lengthdependence of the conduction thereby elucidating the underlying transport mechanisms [3]. Here, we address the influence of laser excitations on the length-dependence of the dc current and the low-frequency noise.

\section{The Wire-Lead Model}

wire-leads model of Fig. 1. It is convenient to separate the contributions from the molecule in the laser field, the ideal leads, and the molecule-leads coupling,

$$
H(t)=H_{\text {molecule }}(t)+H_{\text {leads }}+H_{\text {molecule-leads }} \text {. }
$$

The irradiated molecule is modeled within a tight-binding description taking into account $N$ molecular orbitals $|n\rangle$, which are relevant for the transport. Disregarding the electron-electron interaction, the most general form of the Hamiltonian reads

$$
H_{\text {molecule }}(t)=\sum_{n, n^{\prime}} H_{n n^{\prime}}(t) c_{n}^{\dagger} c_{n^{\prime}}
$$


where the fermionic operators $c_{n}$ and $c_{n}^{\dagger}$ destroy and create, respectively, an electron in the molecular orbital $|n\rangle$. The sums extend over all tight-binding orbitals. The $\mathcal{T}$-periodic time-dependence of the single-particle Hamiltonian $H_{n n^{\prime}}(t)=H_{n n^{\prime}}(t+\mathcal{T})$, reflects the influence of the laser field with frequency $\Omega=2 \pi / \mathcal{T}$. As discussed above, we assume that the leads remain close to equilibrium and hence can be described by grand-canonical ensembles of electrons at temperature $T$ and electro-chemical potential $\mu_{\ell}, \ell=L, R$. Thus, the lead Hamiltonian reads

$$
H_{\text {leads }}=\sum_{\ell q} \epsilon_{\mathrm{q}} c_{\ell q}^{\dagger} c_{\ell q}
$$

where $c_{\ell q}$ destroys an electron in state $q$ in lead $\ell$. All expectation values of lead operators can be traced back to $\left\langle c_{\ell q^{\prime} \ell^{\prime} q^{\prime}}^{\dagger}\right\rangle=\delta_{q q^{\prime}} \delta_{\ell \ell^{\prime}} f\left(\epsilon_{\mathrm{q}}-\mu_{\ell}\right)$, where $f(\epsilon)=\left(1+\mathrm{e}^{\epsilon / k_{B} T}\right)^{-1}$ denotes the Fermi function. The model is completed by the molecule-leads tunneling Hamiltonian

$$
H_{\text {molecule-leads }}=\sum_{\mathrm{q}} V_{L q} c_{L q}^{\dagger} c_{1}+\sum_{\mathrm{q}} V_{R q} c_{R q}^{\dagger} c_{\mathrm{N}}+\text { h.c. },
$$

that connects the left (right) lead to the donor $|1\rangle$ (acceptor $|N\rangle$ ). Since we are not interested here in the effects that arise from the microscopic details of the molecule-lead coupling, we restrict our analysis in the following to energy-independent couplings, i.e., $\Gamma_{\ell}=2 \pi \sum_{q}\left|V_{\ell q}\right|^{2} \delta\left(\epsilon-\epsilon_{\mathrm{q}}\right)=$ const.

\section{Floquet Transport Theory}

For the retarded Green function of the wire electrons, one finds after eliminating the leads the equation of motion [10]

$$
\left[\mathcal{H}(t)-\mathrm{i} \Sigma-\mathrm{i} \hbar \frac{\mathrm{d}}{\mathrm{d} t}\right] G\left(t, t^{\prime}\right)=-\delta\left(t-t^{\prime}\right),
$$

where $\mathcal{H}(t)=\sum_{n, n^{\prime}}|n\rangle H_{n n^{\prime}}(t)\left\langle n^{\prime}\right|$ and $2 \Sigma=|1\rangle \Gamma_{L}\langle 1|+| N\rangle \Gamma_{R}\langle N|$ is the self-energy that results from the coupling to the leads. For the current defined as the change of the charge in the, e.g., left lead, $I_{L}=e(\mathrm{~d} / \mathrm{d} t)\left\langle N_{L}\right\rangle$, we find after some algebra that it assumes the commonly expected "scattering form" [13] but with periodically time-dependent transmission probabilities and, as well, an additional contribution that accounts for a $\mathcal{T}$-periodic charging/discharging of the wire $[10,16]$. Only the former contributes to the time-averaged current

$$
\bar{I}=\frac{e}{2 \pi \hbar} \sum_{k=-\infty}^{\infty} \int \mathrm{d} \epsilon\left\{T_{L R}^{(k)}(\epsilon) f_{R}(\epsilon)-T_{R L}^{(k)}(\epsilon) f_{L}(\epsilon)\right\},
$$

where $T_{L R}^{(k)}(\epsilon)=\Gamma_{L} \Gamma_{R}\left|G_{1 N}^{(k)}(\epsilon)\right|^{2}$ is the transmission of an electron with energy $\epsilon$ from the right lead to the left lead under the absorption (emission) of $|k|$ 
photons if $k>0(k<0)$ and $T_{R L}^{(k)}(\epsilon)$ accordingly. $G_{1 N}^{(k)}(\epsilon)$ denotes the relevant matrix elements of the Fourier transform of the retarded Green function

$$
G^{(k)}(\epsilon)=\int_{0}^{\mathcal{T}} \frac{\mathrm{d} t}{\mathcal{T}} \mathrm{e}^{\mathrm{i} k \Omega t} \int_{-\infty}^{+\infty} \mathrm{d} \tau \mathrm{e}^{\mathrm{i} \epsilon \tau / \hbar} G(t, t-\tau) .
$$

Note that, consistent with Ref. [22], no "Pauli blocking factors" $1-f_{\ell}$ appear in the current formula (6). In contrast to a static situation, this is of relevance here since for a driven system $T_{L R}^{(k)}(\epsilon)$ and $T_{R L}^{(k)}(\epsilon)$ are in general unrelated. Since the coefficients of the equation of motion (5) are $\mathcal{T}$ periodic, a complete solution can be constructed with the help of the Floquet ansatz $\left|\psi_{\alpha}(t)\right\rangle=\exp \left[\left(-\mathrm{i} \epsilon_{\alpha} / \hbar-\gamma_{\alpha}\right) t\right]\left|\Phi_{\alpha}(t)\right\rangle$. The Floquet states $\left|\Phi_{\alpha}(t)\right\rangle=$ $\sum_{k}\left|\Phi_{\alpha k}\right\rangle \exp (-\mathrm{i} k \Omega t)$ obey the time-periodicity of the Hamiltonian and fulfill in a Hilbert space that is extended by a periodic time coordinate the Floquet eigenvalue equation

$$
\left[\mathcal{H}(t)-\mathrm{i} \Sigma-\mathrm{i} \hbar \frac{\mathrm{d}}{\mathrm{d} t}\right]\left|\Phi_{\alpha}(t)\right\rangle=\left(\epsilon_{\alpha}-\mathrm{i} \hbar \gamma_{\alpha}\right)\left|\Phi_{\alpha}(t)\right\rangle .
$$

Since the eigenvalue equation (8) is non-Hermitian, its eigenvalues $\epsilon_{\alpha}-\mathrm{i} \hbar \gamma_{\alpha}$ are generally complex valued and the (right) eigenvectors are not mutually orthogonal. Therefore, we need to solve also the adjoint Floquet equation yielding again the same eigenvalues but providing the adjoint eigenvectors $\left|\Phi_{\alpha}^{+}(t)\right\rangle$. Thus, we find for $\tau>0$ the retarded Green function

$$
G(t, t-\tau)=-\frac{\mathrm{i}}{\hbar} \sum_{\alpha}\left|\psi_{\alpha}(t)\right\rangle\left\langle\psi_{\alpha}^{+}(t-\tau)\right| \Theta(\tau)=G(t+\mathcal{T}, t+\mathcal{T}-\tau)
$$

and, consequently,

$$
G_{n n^{\prime}}^{(k)}(\epsilon)=\sum_{\alpha, k^{\prime}} \frac{\left\langle n \mid \Phi_{\alpha, k^{\prime}+k}\right\rangle\left\langle\Phi_{\alpha, k^{\prime}}^{+} \mid n^{\prime}\right\rangle}{\epsilon-\left(\epsilon_{\alpha}+k^{\prime} \hbar \Omega-\mathrm{i} \hbar \gamma_{\alpha}\right)} .
$$

The current noise is given by the symmetrized auto-correlation function $S_{L}\left(t, t^{\prime}\right)=\frac{1}{2}\left\langle\Delta I_{L}(t) \Delta I_{L}\left(t^{\prime}\right)+\Delta I_{L}\left(t^{\prime}\right) \Delta I_{L}(t)\right\rangle$ of the current fluctuation operator $\Delta I_{L}(t)=I_{L}(t)-\left\langle I_{L}(t)\right\rangle$. It can be shown that after the decay of all transients, $S_{L}\left(t, t^{\prime}\right)=S_{L}\left(t+\mathcal{T}, t^{\prime}+\mathcal{T}\right)$ shares the time-periodicity of the driving. Therefore, it is possible to characterize the noise level by the timeaveraged zero-frequency noise, $\bar{S}_{L}=\int \mathrm{d} \tau \int_{0}^{\mathcal{T}} \mathrm{d} t S_{L}(t, t-\tau) / \mathcal{T}$. Since the total charge is conserved, we find $\bar{S}_{L}=\bar{S}_{R}=\bar{S}$, where $[10,16]$

$$
\begin{array}{r}
\bar{S}=\frac{e^{2} \Gamma_{L} \Gamma_{R}}{2 \pi \hbar} \sum_{k} \int \mathrm{d} \epsilon\left\{\Gamma_{L} \Gamma_{\mathrm{R}}\left|\sum_{k^{\prime}} G_{N 1}^{\left(k^{\prime}-k\right)}\left(\epsilon_{k}\right)\left[G_{N 1}^{\left(k^{\prime}\right)}(\epsilon)\right]^{*}\right|^{2} f_{L}(\epsilon) \bar{f}_{L}\left(\epsilon_{k}\right)\right. \\
\left.+\left|\Gamma_{L} \sum_{k^{\prime}} G_{1 N}^{\left(k^{\prime}-k\right)}\left(\epsilon_{k}\right)\left[G_{11}^{\left(k^{\prime}\right)}(\epsilon)\right]^{*}-\mathrm{i} G_{1 N}^{(-k)}\left(\epsilon_{k}\right)\right|^{2} f_{\mathrm{L}}(\epsilon) \bar{f}_{\mathrm{R}}\left(\epsilon_{k}\right)\right\}
\end{array}
$$

+ same terms with the replacement $(L, 1) \leftrightarrow(R, N)$, 
with $\bar{f}_{L / R}=1-f_{L / R}$ and $\epsilon_{k}=\epsilon+k \hbar \Omega$. In order to characterize the relative noise level, we employ the so-called Fano factor $F=\bar{S} / e|\bar{I}|$ [23]. Expressions (6) and (11) contain as special cases prior findings: In the absence of any driving, the Floquet eigenvalues $\epsilon_{\alpha}-i \hbar \gamma_{\alpha}$ reduce to the complex-valued eigenenergies; this implies $G_{n n^{\prime}}^{(k)}=0$ for all $k \neq 0$, yielding the transmission probability for an electron with energy $E$ of $T(E)=\Gamma_{L} \Gamma_{R}\left|G_{N 1}^{(0)}(E)\right|^{2}$. Thus, the current and the noise in the static limit become

$$
\begin{aligned}
I_{0}=\frac{e}{2 \pi \hbar} \int \mathrm{d} E & T(E)\left[f_{R}(E)-f_{L}(E)\right] \\
S_{0}=\frac{e^{2}}{2 \pi \hbar} \int \mathrm{d} E & \left\{T(E)\left[f_{L}(E)\left[1-f_{L}(E)\right]+f_{R}(E)\left[1-f_{R}(E)\right]\right]\right. \\
+ & \left.T(E)[1-T(E)]\left[f_{R}(E)-f_{L}(E)\right]^{2}\right\}
\end{aligned}
$$

respectively [24]. In order to achieve for the noise an expression that depends only on the transmission probability $T(E)$, we have simplified the second line by use of the relation $\left|\Gamma_{L}(\epsilon) G_{11}(\epsilon)+i\right|^{2}=1-T(\epsilon)$ valid for undriven conductors [13]. Note that by contrast, in the time-dependent case, the noise expression (11) cannot be brought into such a convenient form and, thus in general, still depends on the phases of the propagator.

\section{Conduction Properties of Driven Molecular Bridges}

As a working model we consider a molecule consisting of a donor and an acceptor site and $N-2$ sites in between (cf. Fig. 1). Each of the $N$ sites is coupled to its nearest neighbors by a hopping matrix element $\Delta$. The laser field renders each level oscillating in time with a position-dependent amplitude. The time-dependent molecule Hamiltonian is

$$
\begin{aligned}
H_{n n^{\prime}}(t)= & \Delta \sum_{n=1}^{N-1}(|n\rangle\langle n+1|+| n+1\rangle\langle n|)+E_{1}|1\rangle\langle 1| \\
& +E_{B} \sum_{n=2}^{N-1}|n\rangle\left\langle n\left|+E_{N}\right| N\right\rangle\left\langle N\left|+A \cos (\Omega t) \sum_{n} x_{n}\right| n\right\rangle\langle n|
\end{aligned}
$$

where $x_{n}=(N+1-2 n) / 2$ is the scaled position of site $|n\rangle$. The energy $A$ equals the electron charge multiplied by the electrical field amplitude of the laser and the distance between two neighboring sites. The energies of the donor and the acceptor orbitals, $|1\rangle$ and $|N\rangle$, are assumed to be at the level of the chemical potentials of the attached leads, $E_{1}=E_{N} \approx \mu_{L} \approx \mu_{R}$. The bridge levels $E_{n}, n=2, \ldots, N-1$, lie $E_{B} \gg \Delta$ above the chemical potential.

Below, we will evaluate the current and the noise for zero temperature and use a symmetric coupling, $\Gamma_{L}=\Gamma_{R}=\Gamma$. The hopping matrix element 
$\Delta$ serves as the energy unit; in a realistic wire molecule, $\Delta$ is of the order $0.1 \mathrm{eV}$. Thus, our chosen wire-lead hopping rate $\Gamma=0.1 \Delta$ yields $e \Gamma / \hbar=$ $2.56 \times 10^{-5}$ Ampère and $\Omega \approx 10 \Delta / \hbar$ corresponds to a laser frequency in the near infrared. For a typical distance of $5 \AA$ between two neighboring sites, a driving amplitude $A=\Delta$ is equivalent to an electrical field strength of $2 \times 10^{6} \mathrm{~V} / \mathrm{cm}$.

Let us first discuss the static problem in the absence of the field, i.e. for $A=0$. In the present case where the coupling between two neighboring sites is much weaker than the bridge energy, $\Delta \ll E_{B}$, one finds two types of eigenstates: One group of states is located on the bridge. It consists of $N-2$ levels with energies in the range $\left[E_{B}-2 \Delta, E_{B}+2 \Delta\right]$. In the absence of the driving field, these bridge states mediate the super-exchange between the donor and the acceptor. The two remaining states form a doublet whose states are approximately given by $(|1\rangle \pm|N\rangle) / \sqrt{2}$. Its splitting can be estimated in a perturbational approach [25] and is approximately given by $2 \Delta\left(\Delta / E_{B}\right)^{N-2}$. Thus, the wire can be reduced to a two-level system with the effective tunnel matrix element $\Delta_{D A}=\Delta \exp (-\kappa(N-2))$, where $\kappa=\ln \left(E_{B} / \Delta\right)$. If the chemical potentials of the leads are such that $\mu_{L}>E_{D}$ and $\mu_{R}<E_{A}$, i.e., for a sufficiently large voltage, the current (12) is dominated by the total transmission and for $\Gamma \gg \Delta_{D A}$ can be evaluated to read

$$
I_{0}=\frac{2 e|\Delta|^{2}}{\Gamma} \mathrm{e}^{-2 \kappa(N-2)} .
$$

For the explicit calculation see, e.g., Ref. [26]. In particular, one finds an exponentially decaying length dependence of the current $[3,12]$. Moreover, in this limit, it is also possible to evaluate explicitly the zero-frequency noise to obtain the Fano factor $F=\bar{S} / e|\bar{I}|=1$. This value has a direct physical interpretation: Since the transmissions of electrons across a large barrier are "rare and uncorrelated events", they obey Poisson statistics and, thus, variance and mean value are equal which translates to a Fano factor of one [23].

\subsection{Resonant Excitations}

The magnitude of the current changes significantly when a driving field with a frequency $\Omega \approx E_{B} / \hbar$ is switched on. The resonant bridge levels merge with the donor and acceptor states to form a Floquet state. This opens a direct transport channel resulting in an enhancement of the electron current.

In order to estimate the magnitude of the current through the resonantly driven wire, we disregard all bridge levels besides the one that is in resonance with the donor and the acceptor. Let us assume that this resonant bridge level $\left|\psi_{B}\right\rangle$ extends over the whole bridge such that it occupies the sites $|2\rangle, \ldots,|N-1\rangle$ with equal probability $1 / \sqrt{N-2}$. Accordingly, the overlap between the bridge level and the donor/acceptor becomes

$$
\left\langle 1\left|H_{\text {molecule }}\right| \psi_{B}\right\rangle=\frac{\left\langle 1\left|H_{\text {molecule }}\right| 2\right\rangle}{\sqrt{N-2}}=\frac{\Delta}{\sqrt{N-2}}
$$


and $\left\langle\psi_{B}\left|H_{\text {molecule }}\right| N\right\rangle$ accordingly, while the resonance condition defines the energy of the bridge level as

$$
\left\langle\psi_{B}\left|H_{\text {molecule }}\right| \psi_{B}\right\rangle=\hbar \Omega
$$

(recall that we have assumed $E_{D}=E_{A}=0$ ).

We apply an approximation in the spirit of the one described in [26] and derive a static effective Hamiltonian that describes the time-dependent system. We start by transforming with the unitary operator

$$
S(t)=\exp \left\{-\mathrm{i} \sum_{n=2}^{N-1}|n\rangle\left\langle n\left|\Omega t-\mathrm{i} \frac{A}{\hbar \Omega} \sum_{n=1}^{N}\right| n\right\rangle\langle n| \sin (\Omega t)\right\} .
$$

Note that $S(t)$ obeys the $\mathcal{T}$-periodicity of the original wire Hamiltonian (14). As a consequence, the transformed wire Hamiltonian

$$
\widetilde{H}_{\text {molecule }}(t)=S^{\dagger}(t) H_{\text {molecule }}(t) S(t)-\mathrm{i} \hbar S^{\dagger}(t) \dot{S}(t)
$$

is $\mathcal{T}$-periodic as well. For $\hbar \Omega \gg \Delta$, we can separate time-scales and average $\widetilde{H}_{\text {molecule }}(t)$ over the driving period. In the subspace spanned by $|1\rangle,\left|\psi_{B}\right\rangle$, and $|N\rangle$, the time-averaged wire Hamiltonian reads

$$
H_{\text {molecule,eff }}=\int_{0}^{\mathcal{T}} \frac{\mathrm{d} t}{\mathcal{T}} \widetilde{H}_{\text {molecule }}(t)=\left(\begin{array}{lll}
0 & b & 0 \\
b & 0 & b \\
0 & b & 0
\end{array}\right),
$$

with the effective tunnel matrix element

$$
b=\frac{J_{1}(A / \hbar \Omega)}{\sqrt{N-2}} \Delta,
$$

and $J_{1}$ the first-order Bessel function of the first kind.

The situation described by the Hamiltonian (20) is essentially the following: The central site $\left|\psi_{B}\right\rangle$ is coupled by matrix elements $b$ to the donor and the acceptor site. Since the latter in turn couple to the external leads with a self energy $\Gamma / 2$, their density of states is

$$
\rho(E)=\frac{1}{\pi} \frac{\Gamma / 2}{E^{2}+\Gamma^{2} / 4} .
$$

Then, the tunneling of the electrons from and to the central site is essentially given by the golden rule rate $w=2 \pi|b|^{2} \rho(0) / \hbar$. Like in the static case, we assume that the chemical potential of the left (right) lead lies above (below) the on-site energy of the donor (acceptor) and that therefore the donor is always occupied while the acceptor is always empty. Then, the rate of electrons tunneling from the central site to the acceptor is given by the golden rule rate times the occupation probability $p$ of the state $\left|\psi_{B}\right\rangle$. Accordingly, 
the rate of electron from the donor to $\left|\psi_{B}\right\rangle$ is given by $w$ times the probability $(1-p)$ to find the central site empty. Consequently, the occupation of the resonant bridge level evolves according to $\dot{p}=w(1-p)-w p$. This equation has the stationary solution $p=1 / 2$ and, thus, for resonant excitations, the dc contribution of the time-dependent current is given by

$$
\bar{I}_{\mathrm{res}}=e w p=e \frac{2 A^{2} \Delta^{2}}{(N-2) \hbar^{3} \Omega^{2} \Gamma} .
$$

Here, we have used for small arguments of the Bessel function the approximation $J_{1}(x) \approx x$. The dc current (23) obeys an intriguing scaling behavior as a function of the wire length: Instead of the exponentially decaying length dependence (15) that has been found for the static case, in the presence of resonant driving, a scaling $\bar{I} \propto 1 / N$ emerges. In particular for longer wires, this means that the external field enhances the conductance significantly.

\subsection{Numerical Results}

In order to corroborate the analytical estimates presented above, we treat the transport problem defined by the wire Hamiltonian (14) numerically by solving the corresponding Floquet equation (8) and a subsequent evaluation of the expressions (6) and (11) for the dc current and the zero-frequency noise, respectively. For a wire with $N=5$ sites, one finds peaks in the current when the driving frequency matches the energy difference between the donor/acceptor doublet and one of the $N-2=3$ bridge levels, cf. the solid line in Fig. 2a. The applied voltage is always chosen so small that the bridge levels lie below the chemical potentials of the leads. In Fig. 2a the scale of the abscissa is chosen proportional to $(N-1)$ such that it suggests a common envelope function. Furthermore, we find from Fig. $2 \mathrm{~b}$ that the dc current is proportional to $A^{2} / \Gamma$ provided that $A$ is sufficiently small and $\Gamma$ sufficiently large. The numerical results indicate that the height of the current peaks obeys $\bar{I}_{\text {peak }} \propto \frac{A^{2}}{(N-1) \Gamma}$ which is essentially in accordance with our analytical estimate (23). The main discrepancy comes from the fact that the overlap between the resonant level and the donor/acceptor differs from the estimate (16) by a numerical factor of the order one. Moreover, Fig. 2c demonstrates the at the resonances, the Fano factor assumes values considerably lower than one as expected for the transport through a resonant single level [24].

\section{Conclusions}

We have outlined the Floquet transport formalism which has been derived originally in Refs. [10,16] to provide explicit expressions for the current through time-dependent nanoscale conductors and its zero-frequency noise. With this formalism, the conductance properties of bridged molecular wires 


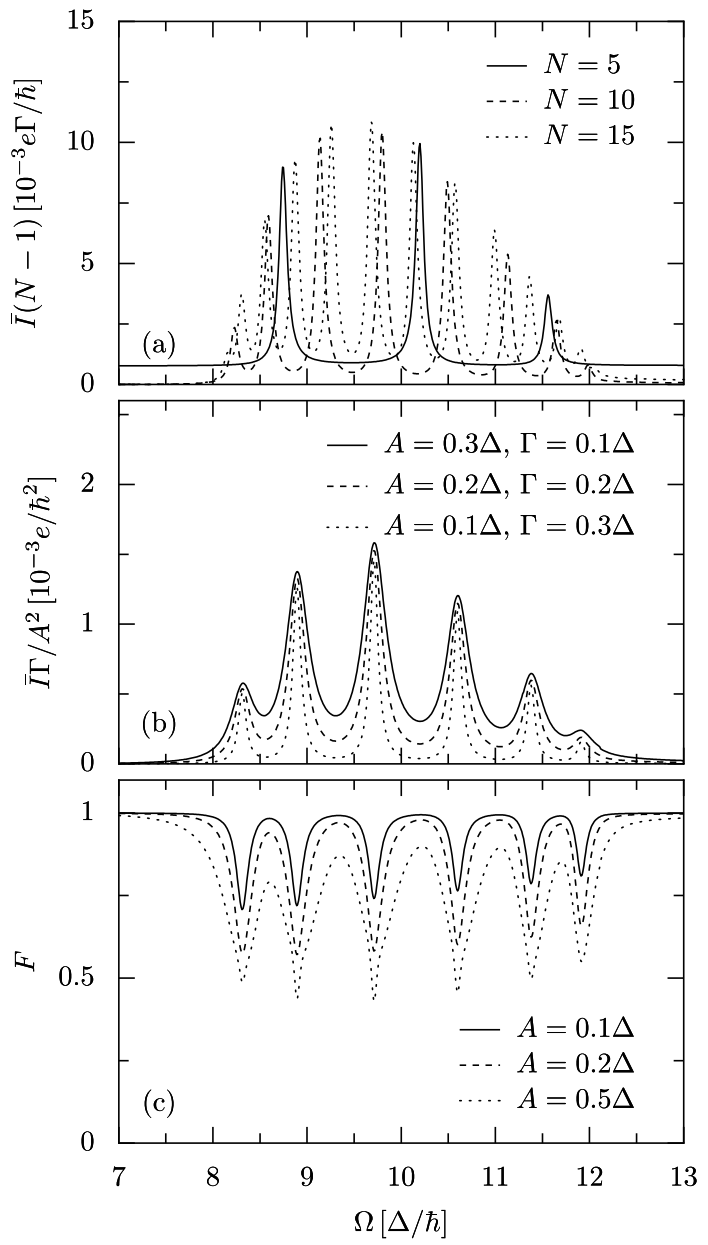

Fig. 2. Exact numerical solution within the Floquet formalism. (a) Average current $\bar{I}$ as a function of the the driving frequency $\Omega$ for various wire length $N$. The scaled amplitude is $A=0.1 \Delta$; the applied voltage $\mu_{R}-\mu_{L}=5 \Delta / e$. The other parameters read $\Gamma=0.1 \Delta, E_{B}=1 \Delta$, and $k_{B} T=0$. (b) Average current for various driving amplitudes $A$ and coupling strengths $\Gamma$ for a wire of length $N=8$. (c) Fano factor $F=\bar{S} / e \bar{I}$ for the wire length $N=8$ and the wire-lead coupling $\Gamma=0.1 \Delta$

have been investigated. This has revealed that resonant excitations from the levels that connect the molecule to the external leads to bridge levels yield peaks in the current as a function of the driving frequency. In a regime with weak driving and weak electron-lead coupling, $\Delta \gg \Gamma, A$, the peak heights scale with the coupling strength, the driving amplitude, and the wire length. The laser irradiation induces a large current enhancement of several orders of magnitude and also can reduce the current noise level. The observation of 
these resonances could serve as an experimental starting point for the more challenging attempt of measuring quantum ratchet effects $[7,8]$ or current switching by laser fields $[9,10]$.

\section{Acknowledgements}

We acknowledge helpful discussions with Sébastien Camalet and Gert-Ludwig Ingold. This work has been supported by the Volkswagen-Stiftung under Grant No. I/77 217 and the DFG Sonderforschungsbereich 486.

\section{References}

1. A. Aviram and M. A. Ratner, Chem. Phys. Lett. 29, 277 (1974).

2. C. Joachim, J. K. Gimzewski, and A. Aviram, Nature 408, 541 (2000).

3. A. Nitzan, Annu. Rev. Phys. Chem. 52, 681 (2001).

4. Special Issue: Processes in Molecular Wires, Vol. 281 of Chem. Phys., edited by P. Hänggi, M. Ratner, and S. Yaliraki (Elsevier, Amsterdam, 2002), pp. $111-502$.

5. X. D. Cui, A. Primak, X. Zarate, J. Tomfohr, O. F. Sankey, A. L. Moore, T. A. Moore, D. Gust, G. Harris, and S. M. Lindsay, Science 294, 571 (2001).

6. J. Reichert, R. Ochs, D. Beckmann, H. B. Weber, M. Mayor, and H. von Löhneysen, Phys. Rev. Lett. 88, 176804 (2002).

7. J. Lehmann, S. Kohler, P. Hänggi, and A. Nitzan, Phys. Rev. Lett. 88, 228305 (2002).

8. J. Lehmann, S. Kohler, P. Hänggi, and A. Nitzan, J. Chem. Phys. 118, 3283 (2003).

9. J. Lehmann, S. Camalet, S. Kohler, and P. Hänggi, Chem. Phys. Lett. 368, $282(2003)$.

10. S. Camalet, J. Lehmann, S. Kohler, and P. Hänggi, Phys. Rev. Lett. 90, 210602 (2003).

11. Y. Meir and N. S. Wingreen, Phys. Rev. Lett. 68, 2512 (1992).

12. V. Mujica, M. Kemp, and M. A. Ratner, J. Chem. Phys. 101, 6849 (1994).

13. S. Datta, Electronic Transport in Mesoscopic Systems (Cambridge University Press, Cambridge, 1995).

14. N. L. Manakov, V. D. Ovsiannikov, and L. P. Rapoport, Phys. Rep. 141, 319 (1986).

15. M. Grifoni and P. Hänggi, Phys. Rep. 304, 229 (1998).

16. S. Camalet, S. Kohler, and P. Hänggi, cond-mat/0402182 (2004).

17. W. B. Davis, M. R. Wasielewski, M. A. Ratner, V. Mujica, and A. Nitzan, J. Phys. Chem. 101, 6158 (1997).

18. D. Segal, A. Nitzan, W. B. Davis, M. R. Wasielewski, and M. A. Ratner, J. Phys. Chem. 104, 3817 (2000).

19. D. Segal and A. Nitzan, Chem. Phys. 281, 235 (2002).

20. V. May, Phys. Rev. B 66, 245411 (2002).

21. D. Segal, A. Nitzan, and P. Hänggi, J. Chem. Phys. 119, 6840 (2003).

22. S. Datta and M. P. Anantram, Phys. Rev. B 45, 13761 (1992).

23. U. Fano, Phys. Rev. 72, 26 (1947). 
24. Ya. M. Blanter and M. Büttiker, Phys. Rep. 336, 1 (2000).

25. M. A. Ratner, J. Phys. Chem. 94, 4877 (1990).

26. S. Kohler, S. Camalet, M. Strass, J. Lehmann, G.-L. Ingold, and P. Hänggi, Chem. Phys. 296, 243 (2004). 